\title{
Crystal structure of potassium antimony hexathiodiphosphate, $\mathrm{KSbP}_{2} \mathrm{~S}_{6}$
}

\author{
V. Manriquez ${ }^{*, I}$, A. Galdámez ${ }^{\mathrm{I}}$, D. Ruiz León ${ }^{\mathrm{I}}$ and M. T. Garland ${ }^{\mathrm{II}}$ \\ I Universidad de Chile, Facultad de Ciencias, Departamento de Química, Casilla 653, Santiago, Chile \\ II Universidad de Chile, Facultad de Ciencias Físicas y Matemáticas, Departamento de Física, Casilla 487-3, Santiago, Chile
}

Received November 18, 2003, accepted and available on-line November 21, 2003, CSD-No. 409751

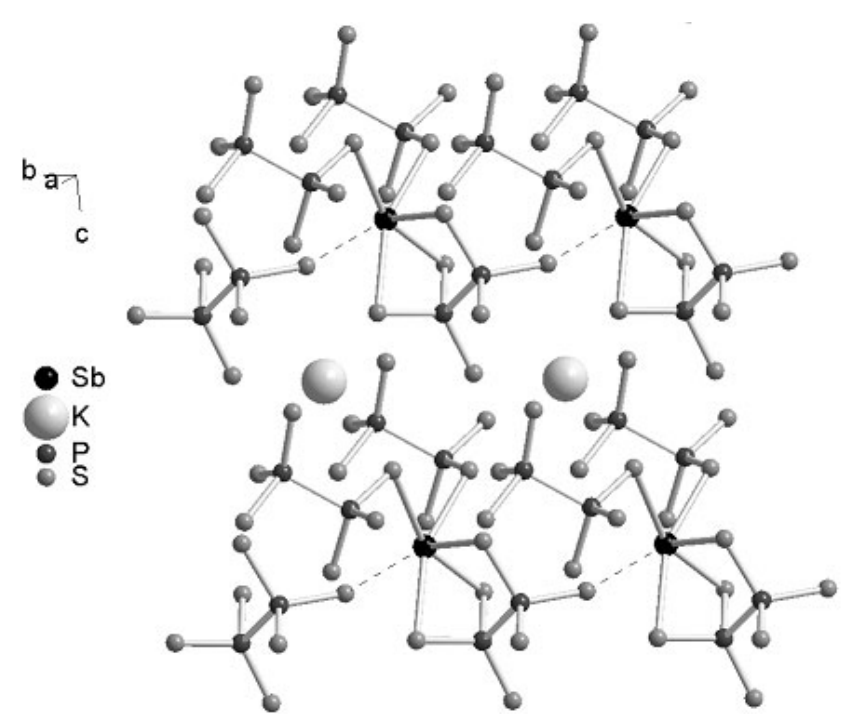

Abstract

$\mathrm{KSbP}_{2} \mathrm{~S}_{6}$, monoclinic, $P 12_{1} 1$ (No. 4), $a=6.605(1) \AA$, $b=7.651(2) \AA, c=9.754(2) \AA, \beta=92.11(3)^{\circ}, V=492.6 \AA^{3}$, $Z=2, R_{\mathrm{gt}}(F)=0.018, w R_{\mathrm{ref}}\left(F^{2}\right)=0.044, T=293 \mathrm{~K}$.

\section{Source of material}

For the synthesis of $\mathrm{KSbP}_{2} \mathrm{~S}_{6}$, the corresponding high purity element powders (99.99\%) supplied by Aldrich were mixed in the stoichiometric amounts, sealed in evacuated quartz tubes, and heated at $1023 \mathrm{~K}$ for two weeks. After the reaction was completed, the sample was slowly cooled to room temperature. All manipulations were carried out under Ar atmosphere

\section{Discussion}

In the course of our work on chalcogen phosphates [1,2], we have preparated the quaternary antimony hexathiodiphosphate $\mathrm{KSbP}_{2} \mathrm{~S}_{6}$. This compound is isostructural to $\mathrm{KBiP}_{2} \mathrm{~S}_{6}$ [3]. As expected, the $\mathrm{Sb}-\mathrm{S}$ bonds in $\mathrm{KSbP}_{2} \mathrm{~S}_{6}$ are shorter that the $\mathrm{Bi}-\mathrm{S}$ bonds in $\mathrm{KBiP}_{2} \mathrm{~S}_{6}$. Also, the smaller radius of $\mathrm{Sb}^{3+}$ results in a decreased coordination number. Thus, each $\mathrm{Sb}^{3+}$ is coordinated by five $\mathrm{S}$ atoms compared with the six-coordinated $\mathrm{Bi}^{3+}$ ions in the isostructural compound. In the title compound, each $\mathrm{Sb}^{3+}$ ion is tri-coordinated by one ethane-like $\left[\mathrm{P}_{2} \mathrm{~S}_{6}\right]^{4-}$ ligand and mono-coordinated by two $\left[\mathrm{P}_{2} \mathrm{~S}_{6}\right]^{4-}$ groups forming chains, while a sixth $\mathrm{S}$ atom connect the $\mathrm{Sb}^{3+}$ to a neighboring chain, through a $\mathrm{Sb}-\mathrm{S}$ contact of 3.4187(9) $\AA$, to form a layer in the $b c$ plane. The layer are separated by the $\mathrm{K}^{+}$ions. The bond lengths $d(\mathrm{Sb}-\mathrm{S})$ range from 2.545(1) $\AA$ to 3.1471(5) $\AA$. The compounds $\mathrm{KMP}_{2} \mathrm{~S}_{6}$ $(\mathrm{M}=\mathrm{Sb}, \mathrm{Bi})$ are structurally related to $\mathrm{Na}_{0.16} \mathrm{Bi}_{1.28} \mathrm{P}_{2} \mathrm{~S}_{6}[4]$ and $\beta-\mathrm{KMP}_{2} \mathrm{Se}_{6}[5]$.
Table 1. Data collection and handling.

$\begin{array}{ll}\text { Crystal: } & \text { yellow block, size } \\ & 0.089 \times 0.104 \times 0.224 \mathrm{~mm} \\ \text { Wavelength: } & \text { Mo } K_{\alpha} \text { radiation }(0.71073 \AA) \\ \mu: & 47.43 \mathrm{~cm}^{-1} \\ \text { Diffractometer, scan mode: } & \text { Bruker AXS SMART CCD }, \varphi / \omega \\ 2 \theta_{\max }: & 55.84^{\circ} \\ N(h k l)_{\text {measured }}, N(h k l)_{\text {unique }}: & 4075,2078 \\ \text { Criterion for } I_{\mathrm{obs}}, N(h k l)_{\mathrm{gt}}: & I_{\mathrm{obs}}>2 \sigma\left(I_{\mathrm{obs}}\right), 2037 \\ N(\text { param })_{\text {refined }}: & 91 \\ \text { Programs: } & \text { SHELXL-97 [6], DIAMOND [7] }\end{array}$

Table 2. Atomic coordinates and displacement parameters (in $\AA^{2}$ ).

\begin{tabular}{lllllllrrrr}
\hline Atom & Site & $x$ & $y$ & $z$ & $U_{11}$ & $U_{22}$ & $U_{33}$ & $U_{12}$ & $U_{13}$ & $U_{23}$ \\
\hline $\mathrm{Sb}$ & $2 a$ & $0.81740(3)$ & $0.76783(2)$ & $0.98199(2)$ & $0.0247(1)$ & $0.0193(1)$ & $0.0235(1)$ & $-0.0014(1)$ & $-0.00049(9)$ & $-0.0024(1)$ \\
$\mathrm{K}$ & $2 a$ & $0.6955(1)$ & $1.0534(1)$ & $0.50699(9)$ & $0.0300(5)$ & $0.0380(5)$ & $0.0314(5)$ & $-0.0002(4)$ & $-0.0020(4)$ & $-0.0003(4)$ \\
$\mathrm{P}(1)$ & $2 a$ & $0.6923(1)$ & $0.3492(1)$ & $0.82655(9)$ & $0.0176(5)$ & $0.0174(4)$ & $0.0167(4)$ & $-0.0019(4)$ & $-0.0007(3)$ & $0.0009(4)$ \\
$\mathrm{P}(2)$ & $2 a$ & $0.7957(1)$ & $0.5678(1)$ & $0.69610(9)$ & $0.0191(4)$ & $0.0199(4)$ & $0.0172(4)$ & $-0.0019(4)$ & $-0.0001(3)$ & $0.0016(4)$ \\
$\mathrm{S}(1)$ & $2 a$ & $0.6716(1)$ & $0.4642(1)$ & $1.01839(8)$ & $0.0297(5)$ & $0.0219(4)$ & $0.0174(5)$ & $-0.0062(4)$ & $0.0032(4)$ & $-0.0019(4)$ \\
$\mathrm{S}(2)$ & $2 a$ & $0.9110(1)$ & $0.1592(1)$ & $0.8275(1)$ & $0.0230(5)$ & $0.0213(4)$ & $0.0247(5)$ & $0.0034(4)$ & $-0.0038(4)$ & $-0.0015(4)$ \\
$\mathrm{S}(3)$ & $2 a$ & $0.4285(1)$ & $0.2626(2)$ & $0.74772(8)$ & $0.0194(4)$ & $0.0263(4)$ & $0.0233(4)$ & $-0.0057(5)$ & $-0.0035(3)$ & $0.0011(5)$ \\
$\mathrm{S}(4)$ & $2 a$ & $1.0547(1)$ & $0.6438(1)$ & $0.8062(1)$ & $0.0180(4)$ & $0.0297(4)$ & $0.0232(5)$ & $-0.0036(4)$ & $0.0002(4)$ & $-0.0025(4)$ \\
$\mathrm{S}(5)$ & $2 a$ & $0.5878(1)$ & $0.7546(2)$ & $0.73068(9)$ & $0.0251(4)$ & $0.0226(4)$ & $0.0268(4)$ & $0.0045(5)$ & $-0.0041(3)$ & $-0.0007(5)$ \\
$\mathrm{S}(6)$ & $2 a$ & $0.8271(2)$ & $0.4844(2)$ & $0.5078(1)$ & $0.0308(6)$ & $0.0367(6)$ & $0.0184(5)$ & $-0.0037(5)$ & $0.0023(4)$ & $-0.0030(4)$ \\
& & & & & & & & & &
\end{tabular}

\footnotetext{
* Correspondence author (e-mail: vmanriqu@uchile.cl)
} 
Acknowledgments. This work was supported by FONDECYT through operating grants No. 1020683 and "Beca Apoyo Tesis CONICYT 2002" and by the Fundación Andes with a single crystal diffractometer.

\section{References}

1. Ruiz-León, D; Manriquez, V.; Kasaneva, J.; Avila, R. E.: Insertion of trivalent cations in layered $\mathrm{MPS}_{3}(\mathrm{M}=\mathrm{Mn}, \mathrm{Cd})$ materials. Mater. Res. Bull. 37 (2002) 981-989.

2. Galdámez, A.; Manríquez, V.; Kasaneva, J.; Avila, R. E.: Synthesis, characterization and electrical properties of quaternary selenodiphosphates: $\mathrm{AMP}_{2} \mathrm{Se}_{6}$ with $\mathrm{A}=\mathrm{Cu}, \mathrm{Ag}$ and $\mathrm{M}=\mathrm{Bi}$, Sb. Mater. Res. Bull. 38 (2003) 1063-1072.

3. Manríquez, V.; Galdámez, A.; Ruiz León, D.; Garland, M. T.; Jiménes, M.: Crystal structure of Potassium Bismuth hexathiodiphosphate $\mathrm{KBiP}_{2} \mathrm{~S}_{6}$. Z. Kristallogr. NCS 218 (2003) 151-152.
4. McCarthy, T.; Kanatzidis, M.: Synthesis in molten alkali metal polythiophosphate fluxes. The new quaternary bismuth and antimony thiophospates $\mathrm{ABiP}_{2} \mathrm{~S}_{7}(\mathrm{~A}=\mathrm{K}, \mathrm{Rb}), \mathrm{A}_{3} \mathrm{M}\left(\mathrm{PS}_{4}\right)_{2}(\mathrm{~A}=\mathrm{K}, \mathrm{Rb}, \mathrm{Cs} ; \mathrm{M}=\mathrm{Sb}, \mathrm{Bi})$, $\mathrm{Cs}_{3} \mathrm{Bi}_{2}\left(\mathrm{PS}_{4}\right)$ and $\mathrm{Na}_{0.16} \mathrm{Bi}_{1.28} \mathrm{P}_{2} \mathrm{~S}_{6}$. J. Alloys Compds. 236 (1996) 70-85.

5. Breshears, J.; Kanatzidis, $\mathrm{M} .: \beta-\mathrm{KBiP}_{2} \mathrm{Se}_{6}(\mathrm{M}=\mathrm{Sb}, \mathrm{Bi})$ : Kinetically Accessible Phases Obtained from rapid crystallization of amorphous precursors. J. Am. Chem. Soc. 122 (2000) 7839-7840.

6. Sheldrick, G. M.: SHELXL-97. Program for crystal structure refinement. University of Göttingen, Germany 1997.

7. Brandenburg, K.: Diamond Version 2.1b, Crystal Impact GbR, Copyright 1996-1999. 